\title{
Oxalate Nephropathy in a Patient with COVID-19- Infection: Causation or Association
}

\section{Lakshmi Kannan ( $\triangle$ lakshmikannan321@gmail.com )}

Pikeville Medical Center https://orcid.org/0000-0001-8306-6723

\section{Rishi Raj}

Pikeville Medical center https://orcid.org/0000-0002-4151-3246

Sheharyar Abbasi

University of Pikeville Kentucky college of Osteopathic Medicine

Aasems Jacob

Pikeville Medical Center https://orcid.org/0000-0002-7783-3786

\section{Case Report}

Keywords: Calcium oxalate, COVID-19, Kidney biopsy, acute kidney injury

Posted Date: February 21st, 2022

DOI: https://doi.org/10.21203/rs.3.rs-1375836/v1

License: (9) This work is licensed under a Creative Commons Attribution 4.0 International License. Read Full License 


\section{Abstract}

The pandemic due to Severe Acute Respiratory Syndrome Coronavirus-2 (SARS-COV2) has led to significant morbidity and mortality over the last two years. Although lungs are the primarily involved organ with infection due to COVID-19, there have been rising rates of COVID-19 related kidney disease. Kidney injury among patients with COVID-19 can present in multiple ways, and different pathophysiologic mechanisms have been suggested. We describe a case of an elderly woman who developed acute kidney injury secondary to oxalate nephropathy in the setting of severe COVID-19 infection and needed hemodialysis. Our case illustrates that calcium oxalate crystal deposition in the kidney (oxalate nephropathy) could be one of the possible pathophysiologic mechanisms of renal dysfunction in the setting of COVID-19.

\section{Introduction}

Renal Dysfunction is frequent in patients with coronavirus disease-19 (COVID-19), although the primary manifestation has been respiratory. Common forms of renal impairment among patients with COVID-19 infection are acute kidney injury (AKI), hematuria, and proteinuria [1]. The pathophysiology of renal dysfunction/AKI remains to be fully elucidated. Due to legitimate concerns about the risk of infection and limited personal protective equipment at the beginning of the pandemic, only limited data is available from live kidney biopsies. However, with the data that is available from post-mortem examinations or autopsies, acute tubular injury (ATI) is found to be the most common pathology [2] followed by collapsing glomerulopathy and thrombotic microangiopathy (TMA) [3].

Various mechanisms have been proposed for kidney injury in COVID-19 including AKI from pulmonary dysfunction/ acute respiratory distress syndrome (ARDS), ischemia, proinflammatory cytokines, direct infection of the renal parenchyma [4], secondary bacterial infection/ sepsis, toxicity of drugs used to treat sepsis and COVID-19 like monoclonal antibodies. However, renal dysfunction secondary to intratubular crystal deposition either as a direct consequence of or from drugs as part of therapeutic intervention for COVID-19 is uncommon. We describe a case of an elderly patient with AKI and COVID-19 infection, who was found to have calcium oxalate crystal deposition on kidney biopsy

\section{Patient Information}

An 82-year-old White woman was transferred from an outside facility for septic shock from Klebsiella pneumonia urinary tract infection, rectal bleeding, and acute hypoxic respiratory failure. Two weeks prior to presentation, she was diagnosed with methicillin-resistant Staphylococcus aureus (MRSA) pneumonia and treated with linezolid. Her past medical history was significant for hypertension, chronic obstructive pulmonary disease (COPD), and atrial fibrillation on anticoagulation with warfarin. She denied any prior history of bariatric surgery. On presentation, vital signs showed blood pressure of 121/41 (mean arterial pressure of 81 ), heart rate of 81 and oxygen saturation of $96 \%$ on $4 \mathrm{~L}$ nasal cannula oxygen. Rest of physical examination was unremarkable. Laboratory workup at presentation showed elevated blood urea 
nitrogen of $54 \mathrm{mg} / \mathrm{dl}$, serum creatinine of $7.7 \mathrm{mg} / \mathrm{dl}$ (compared to the baseline of $0.8-1 \mathrm{mg} / \mathrm{dl}$ ), with a reduced estimated glomerular filtration rate of $4.9 \mathrm{~mL} / \mathrm{min} / 1.73 \mathrm{~m}^{2}$ and hemoglobin of $5.5 \mathrm{~g} / \mathrm{dl}$. Complete laboratory workup on presentation is summarized in Table 1. Screening for COVID - 19 with RT-PCR was positive. A CT chest, abdomen, and pelvis without contrast showed multifocal bilateral diffuse groundglass opacities in the lungs, diffuse enteritis, and extensive diverticulosis. On admission, she received 3 units of packed red blood cell transfusion and was empirically started on broad-spectrum antibiotics with meropenam and linezolid. On hospital day 2 , she had increasing oxygen requirement and eventually needed mechanical ventilation. She was also started on intravenous dexamethasone $6 \mathrm{mg}$ daily and intravenous vitamin C 500 mg daily. On hospital day 3, a colonoscopy was performed which showed multiple polyps which were removed, and pathology showed invasive adenocarcinoma.

Esophagogastroduodenoscopy (EGD) only showed extensive gastritis. Despite improvement in hemodynamics status, patient developed progressive azotemia and oligo-anuria. A renal ultrasound performed was unremarkable. Subsequently, she underwent kidney biopsy on hospital day 5 , she was initiated on intermittent hemodialysis (HD). The timeline of renal function tests and management is described in Fig. 1. Renal biopsy showed global sclerosis of 3 out of 9 glomeruli, presence of acute tubular injury (Fig. 2A) associated with small to large calcium oxalate crystals on both light microscopy and immunofluorescence (Fig. 2B\&C), minimal inflammation without significant eosinophils, mild interstitial fibrosis, and absence of any viral inclusions. Electron microscopy was negative for electrondense deposits. 
Table 1

Laboratory work up on admission

\begin{tabular}{|ll|}
\hline Laboratory test/ Reference Range & Results \\
\hline White blood count (WBCs) (3.0-11.3 K/ul) & 6.10 \\
\hline Red Blood Cell (3.45-5.4 M/ul) & 2.27 \\
\hline Hemoglobin (HGB) (10.0-16.0 gm/dl) & 5.5 \\
\hline Hematocrit (29.9-45.5\%) & 21.4 \\
\hline Platelet (122-454 K/ul) & 65 \\
\hline Sodium (133-144 mmol/L) & 140 \\
\hline Potassium (3.6-5.2 mmol/L) & 5.1 \\
\hline Chloride (98-107 mmol/L) & 107 \\
\hline CO2 (21-32 mmol/L) & 22 \\
\hline Calcium (8.5-10.1mg/dL) & 7.5 \\
\hline Magnesium (1.7-2.4 mg/dL) & 2.3 \\
\hline Phosphorous (2.5-4.9 mg/dL) & 8.1 \\
\hline Blood Urea Nitrogen (7-18 mg/dL) & 54 \\
\hline Creatinine (0.60-1.30 mg/dL) & 7.7 \\
\hline Glomerular Filtration Rate (60-200 mL/min/1.73m²) & 4.9 \\
\hline Glucose (70-110 mg/dL) & 87 \\
\hline Urine Protein (negative mg/dL) & 30 \\
\hline Urine Nitrite (negative) & Negative \\
\hline Urine Leukocytes (negative) & 1.017 \\
\hline Urine Specific Gravity (1.006-1.035) & $20-30$ \\
\hline Urine Blood (negative) & \\
\hline Urine White Blood Cells (WBC) (0.5) & \\
\hline
\end{tabular}

\section{Follow-up And Outcome}

The patient was dialysis dependent throughout her hospitalization. She also developed candidemia and died on hospital day 23. 


\section{Discussion}

Critically ill patients, in general, are prone to develop AKI for varied reasons. In the COVID-19 era, where there are many unknowns, kidney injury could develop from relative hypoperfusion of the kidneys from sepsis/ shock, alteration in microcirculation from cytokine storm, direct tubular epithelial cell damage, hypoxemia, and systemic acidosis which influence kidney vascular resistance and direct or indirect toxicity from medications [5].

In this case, the patient had a clinical picture that was compatible with multifactorial AKI. Nevertheless, the failure to recover kidney function after the initial insult together with the uncertainty related to possible direct viral injury in the kidney prompted histologic examination. The kidney biopsy specimen showed acute tubular injury with no glomerular involvement. Interestingly, the tubular injury was associated with calcium oxalate crystal deposition suggestive of oxalate nephropathy.

Given the biopsy finding of oxalate nephropathy, we speculated that it could be mostly related to secondary hyperoxaluria rather than primary hyperoxaluria as the patient did not have prior history of renal stones. Secondary hyperoxaluria is typically caused by excessive dietary intake of oxalate, Vitamin $\mathrm{C}$, following gastric bypass surgery or small bowel resection, or in presence of underlying conditions such as Crohn's disease or chronic pancreatitis [6].

Based on the study by Marieke et al. on MDCK-I (Madin-Darby canine kidney) cells it was postulated that calcium oxalate crystals do not reach supersaturation in the proximal tubules [7] but reaches supersaturation levels later in the nephron segments as oxalate concentration increases by its tubular secretion and water reabsorption. Also, evidence showed that during the healing phase/ tissue repair and remodeling stage of the tubular epithelial lining, calcium oxalate crystals bind to hyaluronan-rich zones and get internalized which eventually leads to nephrocalcinosis [8].

Given the clinical presentation of our patient, we speculate that the initial tubular damage caused by ischemia from septic shock, severe anemia from GI bleeding, and hypoxemia from ARDS led to acute tubular injury which in turn provided a milieu for calcium oxalate crystals to get deposited. The oxalate is possibly obtained from Vitamin $\mathrm{C}$ administration even though it was only given in very small concentrations (500mg every day for 5 days until the kidney biopsy for a total dose of $2.5 \mathrm{~g}$ ) which is much less compared to other case studies that are studied with COVID and calcium oxalate crystal deposition $[9,10]$. Other possible etiologies for oxalate nephropathy are enteritis which could have contributed to intestinal microbiota alterations and oxalate hyperabsorption and/or direct infection of the kidney by SARS-CoV-2 through angiotensin-converting enzyme 2 receptor. However, we did not identify any viral inclusions in the biopsy sample to suggest a direct role of SARS-CoV-2 in initiating tubular injury.

Even though there are multiple proven pathophysiologic mechanisms of COVID-induced kidney injury, calcium oxalate nephropathy is one such entity that needs to be further investigated. Our case highlights that high doses of vitamin $\mathrm{C}$ administration may not be the only underlying cause of oxalate nephropathy if there is an initial tubular injury from other causes. Unfortunately, we were not able to measure either the 
serum oxalate levels, vitamin C levels, or urine oxalate levels in our case, which is one of the limitations of our study.

Using Vitamin C as a part of the treatment protocol for patients with COVID-19 should be evaluated and only be used if benefits outweigh risks especially for patients who are prone to develop oxalate nephropathy. If a patient develops renal insufficiency while receiving Vitamin $C$ and no potential causes could be found then it should be discontinued and serum oxalate, vitamin $C$, and urine oxalate levels should be measured. Most importantly, for AKIs that do not improve with standard treatment, timely renal biopsy is important to determine the treatment course targeting the exact etiology.

\section{Declarations}

\section{ACKNOWLEDGMENTS}

The authors would like to acknowledge funding support for the publication of this case report which was provided by the education department at Pikeville Medical Center, KY.

\section{STATEMENT OF ETHICS}

Written informed consent was obtained from the patient for publication of the details of their medical case and any accompanying images. A copy of the written consent is available for review by the Editor-inChief of

this journal on request.

\section{CONFLICTS OF INTEREST}

The authors declare that there is no conflict of interest regarding the publication of this article.

\section{DATA AVAILABILITY STATEMENT}

All data generated or analyzed during this study are included in this article.

\section{FUNDING}

The authors received no financial support for the authorship and/or publication of this article.

\section{AUTHOR CONTRIBUTIONS}

The authors confirm contribution to the paper as follows: Lakshmi Kannan- case report conception, Sheharyar Abbasi- Tabulation, Rishi Raj- Analysis of results, Aasems Jacob- Manuscript review. All authors approved the final version of the manuscript.

\section{References}


1. Guan WJ, Ni ZY, Hu Y, et al.: Clinical Characteristics of Coronavirus Disease 2019 in China. The New England journal of medicine. 2020, 382:1708-1720. 10.1056/NEJMoa2002032

2. Hirsch JS, Ng JH, Ross DW, et al.: Acute kidney injury in patients hospitalized with COVID-19. Kidney Int. 2020, 98:209-218. 10.1016/j.kint.2020.05.006

3. Larsen CP, Bourne TD, Wilson JD, et al.: Collapsing Glomerulopathy in a Patient With COVID-19. Kidney Int Rep. 2020, 5:935-939. 10.1016/j.ekir.2020.04.002

4. Yuki K, Fujiogi M, Koutsogiannaki S: COVID-19 pathophysiology: A review. Clin Immunol. 2020, 215:108427. 10.1016/j.clim.2020.108427

5. Verkoelen CF, Verhulst A: Proposed mechanisms in renal tubular crystal retention. Kidney Int. 2007, 72:13-18. 10.1038/sj.ki.5002272

6. Lumlertgul N, Siribamrungwong M, Jaber BL, et al.: Secondary Oxalate Nephropathy: A Systematic Review. Kidney Int Rep. 2018, 3:1363-1372. 10.1016/j.ekir.2018.07.020

7. Verkoelen CF, Van Der Boom BG, Romijn JC: Identification of hyaluronan as a crystal-binding molecule at the surface of migrating and proliferating MDCK cells. Kidney Int. 2000, 58:1045-1054. 10.1046/j.1523-1755.2000.00262.x

8. Schepers MS, Duim RA, Asselman M, et al.: Internalization of calcium oxalate crystals by renal tubular cells: a nephron segment-specific process? Kidney Int. 2003, 64:493-500. 10.1046/j.15231755.2003.00107.x

9. Fontana F, Cazzato S, Giovanella S, et al.: Oxalate Nephropathy Caused by Excessive Vitamin C Administration in 2 Patients With COVID-19. Kidney Int Rep. 2020, 5:1815-1822. 10.1016/j.ekir.2020.07.008

10. Malhotra V, Magoon S, Troyer DA, et al.: Collapsing Focal Segmental Glomerulosclerosis and Acute Oxalate Nephropathy in a Patient With COVID-19: A Double Whammy. J Investig Med High Impact Case Rep. 2020, 8:2324709620963635. 10.1177/2324709620963635

\section{Figures}




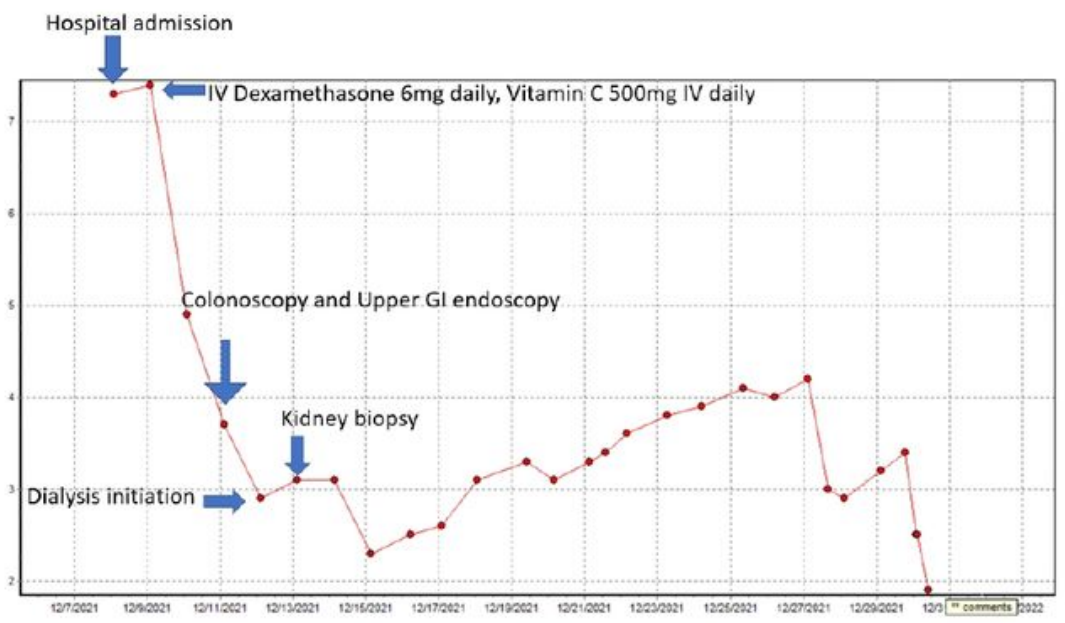

Fig. 1. Timeline of events in relation to creatinine.

\section{Figure 1}

Fig. 1. Timeline of events in relation to creatinine. 
Fig. 2. (A) - Periodic acid- Schiff (PAS) stain showing mild lymphocyte infiltration, loss of proximal tubular brush border consistent with acute tubular injury (ATI). (B, C)- Hematoxylin and Eosin (H\&E) - stained section showing calcium oxalate crystals within the tubules.

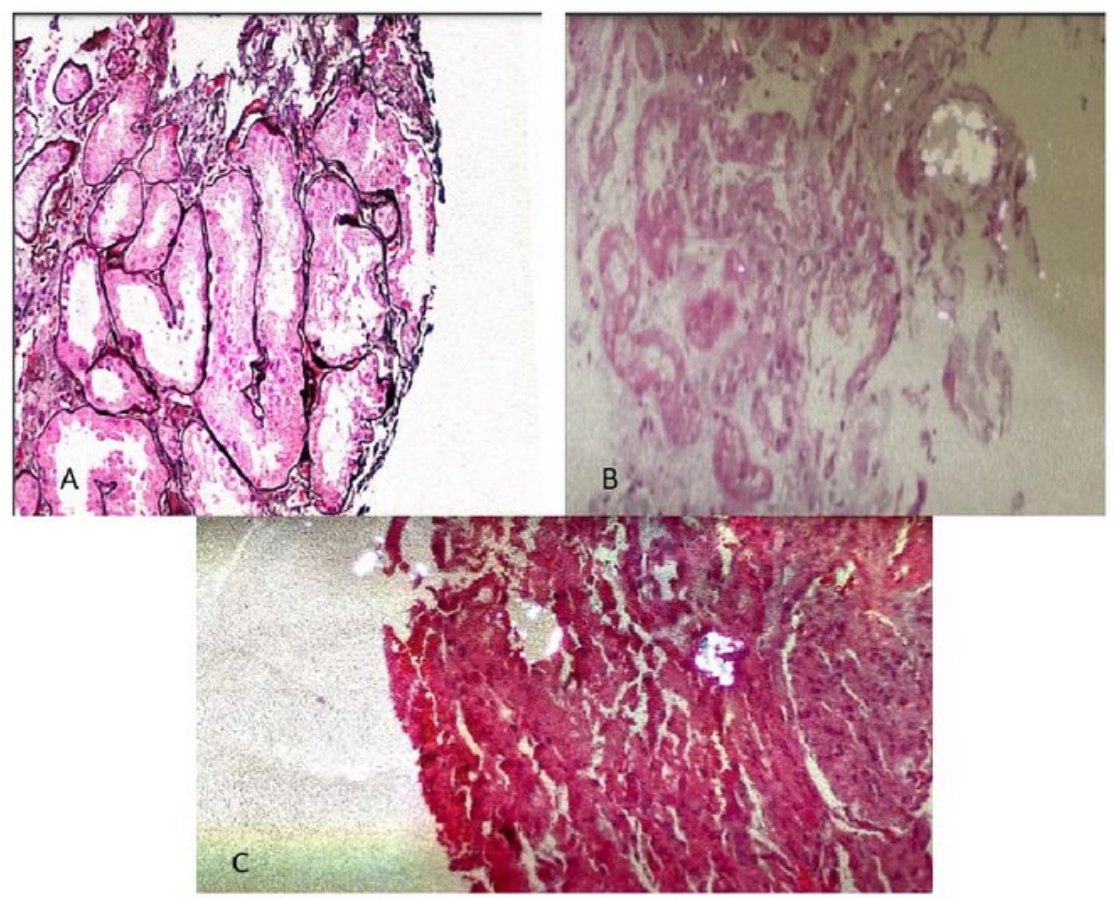

\section{Figure 2}

Fig. 2. (A) - Periodic acid-Schiff (PAS) stain showing mild lymphocyte infiltration, loss of proximal tubular brush border consistent with acute tubular injury (ATI). (B, C)- Hematoxylin and Eosin (H\&E) - stained section showing calcium oxalate crystals within the tubules. 